\title{
Defined Stratification Criterion Permissible Result
}

National Cancer Institute

\section{Source}

National Cancer Institute. Defined Stratification Criterion Permissible Result. NCI

Thesaurus. Code C93418.

A reusable, "template" description of an allowable response to a stratification criterion. 